\title{
Ricci flow on three-dimensional, unimodular metric Lie algebras
}

\author{
David Glickenstein and Tracy L. Payne
}

\begin{abstract}
We give a global picture of the Ricci flow on the space of threedimensional, unimodular, nonabelian metric Lie algebras considered up to isometry and scaling. The Ricci flow is viewed as a two-dimensional dynamical system for the evolution of structure constants of the metric Lie algebra with respect to an evolving orthonormal frame. This system is amenable to direct phase plane analysis, and we find that the fixed points and special trajectories in the phase plane correspond to special metric Lie algebras, including Ricci solitons and special Riemannian submersions. These results are one way to unify the study of Ricci flow on left invariant metrics on three-dimensional, simply-connected, unimodular Lie groups, which had previously been studied by a case-bycase analysis of the different Bianchi classes. In an appendix, we prove a characterization of the space of three-dimensional, unimodular, nonabelian metric Lie algebras modulo isometry and scaling.
\end{abstract}

\section{Introduction}

Ricci flow was introduced by R. Hamilton in [16] to study Riemannian manifolds. In this paper we study the Ricci flow on three-dimensional, unimodular metric Lie algebras. Metric Lie algebras are in one-to-one correspondence with left-invariant Riemannian metrics on simply-connected Lie groups, and Ricci flow on such metrics has been studied by a number of authors (e.g., $[7,12,19,20,22,28,29,33])$. The major advances in this paper are (1) a unification of the trajectories for the Ricci flow, previously viewed individually in case-by-case studies of Bianchi classes, into a single global topological picture, and (2) use of a new technique of flowing the Lie structure constants, which highlights different features of the system than the usual evolution of metric coefficients.

The space of metric Lie algebras has been studied by a number of authors (e.g., $[21,25,28])$. Understanding Ricci flow on the space of metric Lie algebras is important for studying both homogeneous spaces and Ricci flow of general manifolds. A number of Ricci soliton metrics (fixed points of the 
Ricci flow up to diffeomorphism invariance and rescaling) have been found on homogeneous spaces (see, e.g., [1, 15, 24, 26, 28, 32]), and it has been suggested that finding Ricci solitons may be a promising way to attack Alekseevskii's conjecture (see [26, 28]). Lott has shown that three-dimensional, Type III solutions to Ricci flow (as described in [17]) converge to the known homogeneous expanding solitons as they collapse in the limit (see [30]). Ricci flow on homogeneous spaces is also useful in constructing self-dual solutions of Euclidean vacuum Einstein's equations (see [2]).

We will consider the set $\mathcal{M}$ of three-dimensional, nonabelian, unimodular metric Lie algebras modulo isometry and scaling. Milnor gives an excellent description of such metric Lie algebras in [31], in particular showing that there exists a special orthonormal basis $\left\{e_{1}, e_{2}, e_{3}\right\}$ which diagonalizes both the Ricci endomorphism and the Lie bracket (we say that the Lie bracket is diagonalized if $\left[e_{i}, e_{j}\right]$ is a scalar multiple of $\left.e_{i} \times e_{j}\right)$. Thus the set of threedimensional, unimodular metric Lie algebras depends only on three parameters. In fact, there are two natural choices of those three parameters, and the Ricci flow through these parameter spaces takes one of the following forms:

(i) Fix the Lie algebra and let the metric vary.

(ii) Evolve the frame to keep it orthonormal and let the structure constants vary.

In both cases, the Lie bracket and inner product remain diagonal with respect to the frame. However, in the first case the Lie bracket coefficients are fixed and the lengths of basis elements change. In the second case, the Lie bracket coefficients change but the lengths of basis elements do not change (since the basis evolves to stay orthonormal). It is extremely important that the frame remains orthogonal under the flow, which follows from the fact that both the structure constants and the Ricci curvature can always be diagonalized at the same time as the metric. This is true for three-dimensional, unimodular metric Lie algebras, but not in general. The lack of such a frame is the major obstacle for classifying Ricci flow on four-dimensional, simplyconnected homogeneous spaces; Isenberg-Jackson-Lu [20] classify Ricci flow for some Riemannian homogeneous spaces which do admit such a frame.

Elements of the space $\mathcal{M}$ are three-dimensional metric Lie algebras; that is, real vector spaces endowed with inner products. In this category, the most natural notion of normalization is rescaling the inner product by scalar multiplication. Since $\mathcal{M}$ is a three-dimensional space considered modulo rescaling, we have a two-dimensional system of ordinary differential equations (ODEs), which is reasonable to analyze as a dynamical system in the plane. Most previous work on Ricci flow on homogeneous spaces takes the 
first parameterization (e.g., [7, 19, 22]). In contrast, we will take the second parameterization, and consider $\mathcal{M}$ as a quotient of the space of structure constants. This method has previously been used by the second author to study Ricci flow on nilmanifolds [33] (see also [15, 28]). These methods have also been used by researchers in general relativity in studying homogeneous cosmologies (e.g., $[9,10])$.

Let $\phi_{t}$ denote the flow on $\mathcal{M}$ determined by the Ricci flow. Theorem $\mathrm{A}$ describes the topological dynamics of the flow $\phi_{t}$.

Theorem A. The phase space $\mathcal{M}$ is the disjoint union of the following invariant sets (see Figures 1 and 2, and Appendix A for the relationship between $\overline{\mathcal{S}}_{m}, \mathcal{S}_{m}$ and $\left.\mathcal{M}\right)$ :

- four points $p_{1}, p_{2}, p_{3}$ and $p_{4}$;

- six one-dimensional trajectories $T_{1,2}, T_{1,3}, T_{1,3}^{\prime}, T_{1,4}, T_{2,3}$ and $T_{3,4}$; and

- three connected two-dimensional open sets $B_{1,4}, B_{1,3}$ and $B_{1,3}^{\prime}$;

such that

- the points $p_{1}, p_{2}, p_{3}$ and $p_{4}$ are fixed by $\phi_{t}$;

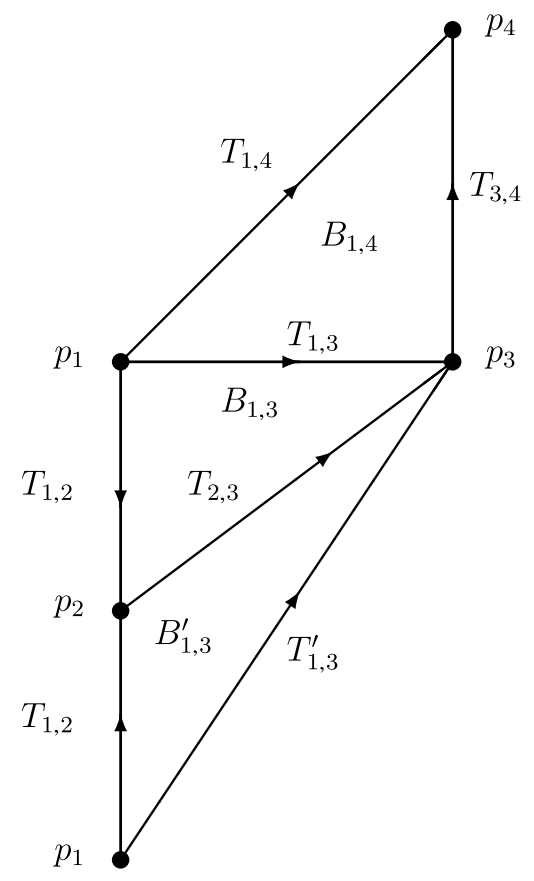

Figure 1: Schematic of $\overline{\mathcal{S}}_{m}$ with Ricci flow lines. 


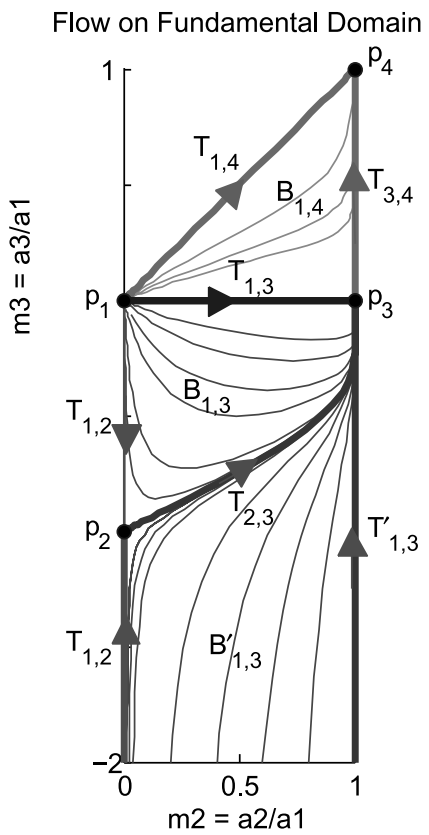

Figure 2: The space $\mathcal{S}_{m}$ with Ricci flow lines.

- the orbit of a point $p$ in a $T_{i, j}$ or $T_{i, j}^{\prime}$ has $\lim _{t \rightarrow-\infty} \phi_{t}(p)=p_{i}$, and $\lim _{t \rightarrow \infty} \phi_{t}(p)=p_{j}$; and

- the orbit of a point $p$ in $B_{i, j}$ or $B_{i, j}^{\prime}$ has $\lim _{t \rightarrow-\infty} \phi_{t}(p)=p_{i}$, and $\lim _{t \rightarrow \infty} \phi_{t}(p)=p_{j}$.

Theorem B interprets Theorem A geometrically. In the sequel, we will say a point in the phase space represents a particular metric, although we actually mean that it represents the homothety class of the metric, i.e., the equivalence class of the metric up to isometry and scaling (see Definition 2.2 and Remark 2.1). Given a Lie group $G$, we will denote the universal covering group by $\tilde{G}$. Furthermore, recall that a Riemannian submersion $f: M \rightarrow N$ is a map such that the differential $f_{*}$ maps $H_{x}$ isometrically to $T_{f(x)} N$, where $H_{x}$ is the orthogonal complement of the kernel of $f_{*}$ (see [3, Chapter 9]).

Theorem B. The decomposition of the phase space $\mathcal{M}$ in Theorem $A$ corresponds geometrically as follows.

(i) Each of the four fixed points $p_{1}, p_{2}, p_{3}$ and $p_{4}$ represents a soliton metric: 
- $p_{1}$ represents the soliton metric on the three-dimensional Heisenberg group $H(3)$.

- $p_{2}$ represents the soliton metric on the three-dimensional solvable group $E(1,1)$.

- $p_{3}$ represents the flat metric on the three-dimensional Euclidean group $\widehat{E(2)}$.

- $p_{4}$ represents the round metric on the group $\mathrm{SU}(2)$.

(ii) The five trajectories $T_{1,2}, T_{1,3}, T_{1,3}^{\prime}, T_{1,4}$, and $T_{3,4}$ have Riemannian submersion structures:

- $T_{1,2}$ consists of left-invariant metrics on $E(1,1)$ (often denoted $\mathrm{Sol}$ ). These metrics fiber as Riemannian submersions over $\mathbb{R}$.

- $T_{1,3}$ consists of left-invariant metrics on $\widetilde{E(2)}$. These metrics fiber as Riemannian submersions over $\mathbb{R}$.

- $T_{1,3}^{\prime}$ consists of left-invariant metrics on $\widehat{\mathrm{SL}_{2}(\mathbb{R})}$ which fiber as Riemannian submersions over the hyperbolic plane $\mathbb{H}^{2}$.

- $T_{1,4}$ and $T_{3,4}$ consist of left-invariant metrics on $\mathrm{SU}(2)$ which fiber as Riemannian submersions over the round sphere $\mathbb{S}^{2}$ (these Riemannian manifolds are often called Berger spheres). The trajectory $T_{1,4}$ corresponds to submersions whose fibers are larger than those of the round three-sphere (corresponding to the point $p_{4}$ ) and the trajectory $T_{3,4}$ corresponds to submersions whose fibers are smaller than those of the round three-sphere.

(iii) The three connected open sets $B_{1,4}, B_{1,3}$ and $B_{1,3}^{\prime}$ have the structures:

- $B_{1,4}$ consists of left-invariant metrics on $\mathrm{SU}(2)$.

- $B_{1,3}$ and $B_{1,3}^{\prime}$ consist of left-invariant metrics on $\widetilde{\mathrm{SL}_{2}(\mathbb{R})}$.

Note that the trajectory $T_{2,3}$ is still somewhat mysterious. This trajectory was discovered independently by Cao et al. [4], and evidence for it was present in [5]. Preliminary evidence suggests that this trajectory is not invariant under cross curvature flow, which may indicate it does not arise from extra symmetries of the Riemannian metric, as the other special orbits do (see Remark 5.2).

In formulating the convergence results, we have implicitly chosen a renormalization of the Ricci flow. These convergence results are related to, but sometimes different than, those in $[12,19,29]$. There is still not a consensus as to the best renormalization method for classifying singularities of the Ricci flow; in the final section of the paper, we discuss this issue, comparing previous work with our own. 
The organization of this paper is as follows. In Section 2, we introduce notation and discuss the space of three-dimensional, unimodular metric Lie algebras and their curvatures. In Section 3 we derive the Ricci flow equations on the space of structure constants. In Section 4 we analyze the dynamics of the Ricci flow equations on a natural phase space and then on $\mathcal{M}$, completing the proof of Theorem A. In Section 5 we analyze the dynamics geometrically, relating fixed points and special trajectories to Ricci solitons and Riemannian submersions, proving Theorem B. In Section 6 we discuss how our convergence results relate to the current literature on Ricci flows on three-dimensional, unimodular metric Lie algebras and Lie groups. Finally, we include an appendix which give the details of characterizing the space $\mathcal{M}$.

\section{Metric Lie algebras and their curvatures}

Consider the following definitions.

Definition 2.1. A metric Lie algebra $(\mathfrak{g}, \mathrm{Q})$ is a Lie algebra $\mathfrak{g}$ together with an inner product $Q$ on $\mathfrak{g}$. The dimension of the metric Lie algebra is the dimension of $\mathfrak{g}$, and it is unimodular or nonabelian if the Lie algebra $\mathfrak{g}$ is unimodular (i.e., $\operatorname{ad}_{X}$ is trace free for all $X \in \mathfrak{g}$ ) or nonabelian, respectively.

Recall that there is a one-to-one correspondence between Lie algebras $\mathfrak{g}$ and simply connected Lie groups $G$. Furthermore, there is a one-to-one correspondence between metric Lie algebras $(\mathfrak{g}, \mathrm{Q})$ and simply connected Lie groups $G$ with a left-invariant Riemannian metric $g$. We will use the Riemannian manifold $(G, g)$ corresponding to the metric Lie algebra $(\mathfrak{g}, \mathrm{Q})$ for the following definition.

Definition 2.2. A linear map $L: \mathfrak{g} \rightarrow \mathfrak{g}^{\prime}$ is an isometry of metric Lie algebras $(\mathfrak{g}, \mathrm{Q})$ and $\left(\mathfrak{g}^{\prime}, \mathrm{Q}^{\prime}\right)$ if it is the differential of a Riemannian isometry $(G, g) \rightarrow\left(G^{\prime}, g^{\prime}\right)$ between the corresponding simply connected Lie groups with induced left-invariant metrics. Two metrics $Q$ and $Q^{\prime}$ on $\mathfrak{g}$ are homothetic if $\mathrm{Q}^{\prime}=c \mathrm{Q}$ for some $c>0$. We say $(\mathfrak{g}, \mathrm{Q})$ and $\left(\mathfrak{g}^{\prime}, \mathrm{Q}^{\prime}\right)$ are equivalent up to isometry and scaling if there exists a diffeomorphism $\phi: G \rightarrow G^{\prime}$ and $c>0$ such that $g=c \phi^{*} g^{\prime}$. The space of three-dimensional, nonabelian, unimodular metric Lie algebras modulo isometry and scaling will be denoted by $\mathcal{M}$.

Remark 2.1. A related definition is that of isomorphism between metric Lie algebras. One says two metric Lie algebras $(\mathfrak{g}, \mathbf{Q})$ and $\left(\mathfrak{g}^{\prime}, \mathrm{Q}^{\prime}\right)$ are 
isomorphic if there is a linear map $L: \mathfrak{g} \rightarrow \mathfrak{g}^{\prime}$ which is a Lie algebra isomorphism and satisfies $L^{*} Q^{\prime}=\mathrm{Q}$. The notion of isomorphism in this definition is stronger than the notion of isometry in Definition 2.2. That is, given an isomorphism of metric Lie algebras, one can use the group action to extend this to an isometry of the corresponding simply connected Lie groups with corresponding left-invariant metrics. However, it is possible to have isometries of the groups which are not isomorphisms of metric Lie algebras. For instance, there is a flat metric $g_{0}$ on the Lie group $E(2)$ of Euclidean transformations (as well as its universal cover), and there is a flat metric $g_{1}$ on the abelian group $\mathbb{R}^{3}$. There is an isometry between $\left(\widetilde{E(2)}, g_{0}\right)$ and $\left(\mathbb{R}^{3}, g_{1}\right)$, but this isometry is not a group isomorphism, and so its differential is not an isomorphism of metric Lie algebras.

In the beautiful paper of Milnor [31], geometric properties of leftinvariant metrics on three-dimensional Lie groups are studied in detail. Milnor computes the curvatures of three-dimensional, unimodular metric Lie algebras:

Theorem 2.1 ([31, Theorem 4.3]). Suppose $(\mathfrak{g}, \mathrm{Q})$ is a three-dimensional, unimodular metric Lie algebra. Then there exists a Q-orthonormal frame $\mathcal{B}=\left\{e_{1}, e_{2}, e_{3}\right\}$ for $\mathfrak{g}$ such that Lie brackets for $\mathfrak{g}$ are determined by

$$
\left[e_{2}, e_{3}\right]=a_{1} e_{1}, \quad\left[e_{3}, e_{1}\right]=a_{2} e_{2} \quad \text { and } \quad\left[e_{1}, e_{2}\right]=a_{3} e_{3},
$$

for some constants $a_{1}, a_{2}, a_{3} \in \mathbb{R}$. Furthermore, this basis diagonalizes the Ricci endomorphism Rc such that

$$
[\mathrm{Rc}]_{\mathcal{B}}=2\left[\begin{array}{lll}
\mu_{2} \mu_{3} & & \\
& \mu_{1} \mu_{3} & \\
& & \mu_{1} \mu_{2}
\end{array}\right]
$$

where

$$
\mu_{i}=\frac{1}{2}\left(a_{1}+a_{2}+a_{3}\right)-a_{i}
$$

The sectional curvatures are given by

$$
\begin{aligned}
& K\left(e_{2} \wedge e_{3}\right)=-\mu_{2} \mu_{3}+\mu_{1} \mu_{3}+\mu_{1} \mu_{2}, \\
& K\left(e_{3} \wedge e_{1}\right)=\mu_{2} \mu_{3}-\mu_{1} \mu_{3}+\mu_{1} \mu_{2}, \\
& K\left(e_{1} \wedge e_{2}\right)=\mu_{2} \mu_{3}+\mu_{1} \mu_{3}-\mu_{1} \mu_{2} .
\end{aligned}
$$


Table 1: The three-dimensional, nonabelian, unimodular Lie groups/ algebras.

\begin{tabular}{lll}
\hline $\begin{array}{l}\text { Signs of } \\
\left\{a_{1}, a_{2}, a_{3}\right\}\end{array}$ & $\begin{array}{l}\text { Associated Lie } \\
\text { algebra }\end{array}$ & Associated Lie groups \\
\hline,,+++ & $\mathfrak{s u}(2) \cong \mathfrak{s o}(3)$ & $\mathrm{SU}(2)$ or $\mathrm{SO}(3)=\mathrm{Isom}_{+}\left(\mathbb{S}^{2}\right)$ \\
,,++- & $\mathfrak{s}(2, \mathbb{R})$ & $\mathrm{SL}_{2}(\mathbb{R})$ or $\mathrm{O}(1,2)$ or $\mathrm{Isom}_{+}\left(\mathbb{H}^{2}\right)$ \\
,,++ 0 & $\mathfrak{e}(2)$ & $E(2)=\mathrm{Isom}\left(\mathbb{E}^{2}\right)$ \\
,,+- 0 & $\mathfrak{e}(1,1)$ & $E(1,1)=\mathrm{Sol}$ \\
$+, 0,0$ & $\mathfrak{h}(3)$ & $H(3)=\mathrm{Nil}$ \\
\hline
\end{tabular}

Scalar curvature $\rho$ is

$$
\rho=2\left(\mu_{2} \mu_{3}+\mu_{1} \mu_{3}+\mu_{1} \mu_{2}\right)
$$

Milnor also describes the isomorphism type of a Lie algebra determined by Equations (2.1) based on the signs of $a_{1}, a_{2}, a_{3}$; see Table 1 (where if the signs are all multiplied by -1 , the Lie algebra is the same).

Recalling the space $\mathcal{M}$ of metric Lie algebras from Definition 2.2, according to Theorem 2.1 we have the following lemma.

Lemma 2.1. Let $\tilde{\Psi}:\left(\mathbb{R}^{3} \backslash\{(0,0,0)\}\right) \rightarrow \mathcal{M}$ be the map which takes $\left(a_{1}, a_{2}\right.$, $\left.a_{3}\right)$ to the equivalence class of the metric Lie algebra defined by an orthonormal basis $\left\{e_{1}, e_{2}, e_{3}\right\}$ with Lie bracket determined by (2.1). The map $\tilde{\Psi}$ is surjective.

Notice that we have excluded the point $(0,0,0)$, representing the abelian Lie algebra $\mathbb{R}^{3}$, from the domain of $\tilde{\Psi}$. This will allow $\tilde{\Psi}$ to descend to a map from $\mathbb{R P}^{2}$ to $\mathcal{M}$ so that we can consider metric Lie algebra equivalence up to scaling.

Proof. Certainly the above definition defines a Lie bracket, and Theorem 2.1 shows that every three-dimensional, unimodular Lie algebra can be written in this way. Since $\mathcal{M}$ is a quotient of the set of all such Lie algebras, the result follows.

We would like to describe the space $\mathcal{M}$ using a fundamental domain. Since $\tilde{\Psi}$ descends to a map from $\mathbb{R P}^{2}$ to $\mathcal{M}$, we start with the coordinates 
$\left(m_{2}, m_{3}\right)=\left(\frac{a_{2}}{a_{1}}, \frac{a_{3}}{a_{1}}\right)$ on $\mathbb{R P}^{2}$. Let

$$
\mathcal{S}_{m}=\left\{\left(m_{2}, m_{3}\right) \in \mathbb{R}^{2}: 0 \leq m_{2} \leq 1 \text { and } m_{3} \leq m_{2}\right\},
$$

and let $\sim$ be the equivalence relation on $\mathcal{S}_{m}$ that is determined by

$$
\left(0, m_{3}\right) \sim\left(0,1 / m_{3}\right),
$$

if $m_{3} \neq 0$. Since a fundamental domain should be compact, we need to compactify $\mathcal{S}_{m}$, and so we also introduce the compact set

$$
\overline{\mathcal{S}}_{m}=\mathcal{S}_{m} \cup\{\infty\}
$$

which is given the one-point-compactification topology, i.e., open neighborhoods of $\infty$ consist of the complements of compact subsets of $\mathcal{S}_{m}$. We can extend $\sim$ to an equivalence relation on $\overline{\mathcal{S}}_{m}$ by adding the equivalence

$$
\infty \sim(0,0)
$$

In the appendix, we prove the following:

Theorem 2.2. There is a bijection between $\overline{\mathcal{S}}_{m} / \sim$ and $\mathcal{M}$.

Using the quotient topology on $\overline{\mathcal{S}}_{m} / \sim$, there is a natural topology on $\mathcal{M}$ which makes this map a homeomorphism. Note that this topology is quite natural, as it means that a sequence in $\mathcal{S}_{m}$ converging to infinity must converge to $(0,0)$ in $\overline{\mathcal{S}}_{m} / \sim$. By switching the roles of $a_{1}$ and $a_{3}$, one sees that this convergence is natural in the space of metric Lie algebras. Note that convergence in this setting is equivalent to convergence of structure constants, which implies convergence of the Riemannian connection. By a standard Arzela-Ascoli argument, it implies a subsequence of Riemannian metrics converge in $C^{0}$ (see [8, Chapter 3]). In fact, since all covariant derivatives of curvatures are polynomials in the structure constants, there is a subsequence that converges in $C^{\infty}$. It is not clear in the general case whether one can eliminate passing to the subsequence, although Lauret shows this is the case for nilpotent groups in [27] since the metric can also be written as a polynomial in the structure constants.

The proof of Theorem 2.2, as well as some of discussion in Section 6, requires the covariant derivatives of the Ricci tensor, which can be derived in a straightforward way from the formulas in Theorem 2.1. They also appear in $[23]$. 
Proposition 2.1. Suppose ( $\mathfrak{g}, \mathrm{Q})$ is a three-dimensional, unimodular metric Lie algebra. The covariant derivative of the Ricci operator satisfies

$$
|D \mathrm{Rc}|^{2}=8\left(\left(\mu_{1}-\mu_{3}\right)^{2} \mu_{2}^{4}+\left(\mu_{1}-\mu_{2}\right)^{2} \mu_{3}^{4}+\left(\mu_{2}-\mu_{3}\right)^{2} \mu_{1}^{4}\right),
$$

where $\mu_{i}$ are as in Theorem 2.1.

\section{Ricci deformation of three-dimensional unimodular metric Lie algebras}

We now derive the equations for Ricci flow on $\mathcal{M}$. Recall that on a Riemannian manifold $(M, g)$, the Ricci flow is the solution to the equations

$$
\frac{\partial g}{\partial t}=-2 \operatorname{Rc}(g)
$$

For a left-invariant metric on a Lie group $G$, this flow reduces to a flow of the inner product $\mathrm{Q}$ on the Lie algebra $\mathfrak{g}$ of $G$; that is, it reduces to a flow of metric Lie algebras $\left(\mathfrak{g}, Q_{t}\right)$. Recall that Theorem 2.1 implies that for any inner product $Q$ on a three-dimensional, unimodular Lie algebra, we can find a $\mathbf{Q}$-orthonormal basis $\mathcal{B}=\left\{e_{1}, e_{2}, e_{3}\right\}$ for $\mathfrak{g}$ which diagonalizes the Ricci tensor. We will see two ways to formulate the Ricci flow:

(i) Fix the basis $\overline{\mathcal{B}}=\left\{\bar{e}_{1}, \bar{e}_{2}, \bar{e}_{3}\right\}$ from Theorem 2.1 which is orthonormal with respect to $Q_{0}$, the initial inner product, and consider the evolution of the metric coefficients $\mathrm{Q}_{t}\left(\bar{e}_{i}, \bar{e}_{j}\right)$. In this case, the structure constants $\lambda_{1}, \lambda_{2}, \lambda_{3}$ with respect to the basis $\overline{\mathcal{B}}$, i.e.,

$$
\left[\bar{e}_{2}, \bar{e}_{3}\right]=\lambda_{1} \bar{e}_{1}, \quad\left[\bar{e}_{3}, \bar{e}_{1}\right]=\lambda_{2} \bar{e}_{2} \quad \text { and } \quad\left[\bar{e}_{1}, \bar{e}_{2}\right]=\lambda_{3} \bar{e}_{3},
$$

are fixed in time.

(ii) Evolve the basis $\mathcal{B}_{t}=\left\{e_{1}(t), e_{2}(t), e_{3}(t)\right\}$ to be orthonormal with respect to $Q_{t}$, so that the metric is the identity in this frame, but the structure constants $a_{1}, a_{2}, a_{3}$ with respect to $\mathcal{B}_{t}$, which are of the form (2.1), depend on time.

In general, if $\overline{\mathcal{B}}=\left\{\bar{e}_{1}, \bar{e}_{2}, \bar{e}_{3}\right\}$ is a basis which is orthogonal with respect to a metric $\bar{Q}$ and satisfies (3.1), and

$$
q_{i}=\overline{\mathrm{Q}}\left(\bar{e}_{i}, \bar{e}_{i}\right),
$$


for $i=1,2,3$, then we see that $\mathcal{B}=\left\{e_{1}, e_{2}, e_{3}\right\}$, where $e_{i}=\bar{e}_{i} / \sqrt{q_{i}}$, is orthonormal and the structure constants $a_{i}$ (as in Theorem 2.1) are related by

$$
a_{i}=\sqrt{\frac{q_{i}}{q_{j} q_{k}}} \lambda_{i} .
$$

This is how one can relate the solution flows in formulations (i) and (ii). As first described in [19], in formulation (i) the Ricci curvature is diagonal with respect to the initial metric $Q_{0}$, and so the following Ricci flow evolution can be derived for $q_{1}, q_{2}, q_{3}$ (see formulas from Theorem 2.1):

$$
\begin{aligned}
& \frac{d}{d t} \log q_{1}=-4 \mu_{2} \mu_{3}=-a_{1}^{2}+\left(a_{2}-a_{3}\right)^{2}, \\
& \frac{d}{d t} \log q_{2}=-4 \mu_{1} \mu_{3}=-a_{2}^{2}+\left(a_{3}-a_{1}\right)^{2}, \\
& \frac{d}{d t} \log q_{3}=-4 \mu_{1} \mu_{2}=-a_{3}^{2}+\left(a_{1}-a_{2}\right)^{2},
\end{aligned}
$$

where the $a_{i}$ are explicit functions of the $q_{i}$ (with fixed parameters $\lambda_{i}$ ) defined by (3.2). Thus equations (3.3) form an autonomous system of ODEs in the variables $q_{1}, q_{2}, q_{3}$. Since the fixed basis $\overline{\mathcal{B}}$ is orthogonal with respect to $\mathrm{Q}_{t}$ (as determined by $\left.q_{1}(t), q_{2}(t), q_{3}(t)\right)$ and (3.1) continues to be satisfied at each time $t$, we see that the flow (3.3) really is the Ricci flow for all time. The fact that the flow remains diagonal is a special property of three-dimensional, unimodular metric Lie algebras, and is not true in general (see, e.g., [20]).

Noting that the right sides of the ODEs (3.3) only contain the $a_{i}$, without explicitly containing the $q_{i}$, it is natural to consider Formulation (ii). The evolution equations for $a_{i}$ are easily derived using (3.3) and (3.2). Due to Theorem 2.2 (and the preceding discussion from Section 2), we will also be interested in

$$
\begin{aligned}
& m_{2}(t)=a_{2}(t) / a_{1}(t), \\
& m_{3}(t)=a_{3}(t) / a_{1}(t) .
\end{aligned}
$$

We have the following.

Proposition 3.1. Let $G$ be a simply connected, three-dimensional, nonabelian, unimodular Lie group with left-invariant metric $g$. Then the Ricci flow on $G$ with initial metric $g$ corresponds to a flow of metric Lie algebras $\left(\mathfrak{g}, \mathrm{Q}_{t}\right)$, where $\mathfrak{g}$ is the Lie algebra of $G$ and $\mathrm{Q}_{0}$ is $g$ restricted to $T_{e} G \cong \mathfrak{g}$. This flow can be realized as a flow of structure constants $a_{1}, a_{2}, a_{3}$ (as determined by Theorem 2.1), and, if we suppose that $a_{1} \neq 0$, the ratios $m_{2}$ and 
$m_{3}$ (as defined in (3.4)) obey the equations

$$
\begin{aligned}
& \frac{d m_{2}}{d t}=a_{1}^{2} m_{2}\left(1-m_{2}\right)\left(1+m_{2}-m_{3}\right) \\
& \frac{d m_{3}}{d t}=a_{1}^{2} m_{3}\left(1-m_{3}\right)\left(1-m_{2}+m_{3}\right) .
\end{aligned}
$$

Remark 3.1. We also note that if $a_{1} \neq 0$,

$$
\frac{d}{d t}\left(\log a_{1}\right)=\frac{1}{2}\left[\frac{d}{d t}\left(\log q_{1}\right)-\frac{d}{d t}\left(\log q_{2}\right)-\frac{d}{d t}\left(\log q_{3}\right)\right]=2 K\left(e_{2} \wedge e_{3}\right) .
$$

The reader may be troubled that the expressions for $d m_{2} / d t$ and $d m_{3} / d t$ in the proposition are not solely functions of $m_{2}$ and $m_{3}$; however, they are useful, because we will be interested in imagining slope fields for the flow of $m_{2}$ and $m_{3}$ in the $m_{2}-m_{3}$ plane. The common (positive) term $a_{1}^{2}$ simply affects the speed of motion and not the direction of motion, so the trajectories of $m_{2}, m_{3}$ under the ODEs (3.5) are the same as the trajectories of the autonomous ODEs

$$
\begin{aligned}
& \frac{d m_{2}}{d t}=m_{2}\left(1-m_{2}\right)\left(1+m_{2}-m_{3}\right) \\
& \frac{d m_{3}}{d t}=m_{3}\left(1-m_{3}\right)\left(1-m_{2}+m_{3}\right) .
\end{aligned}
$$

Proof. The translation to the metric Lie algebra was described at the beginning of this section. Observe that

$$
m_{2}=\frac{a_{2}}{a_{1}}=\frac{\lambda_{2}}{\lambda_{1}} \sqrt{\frac{q_{2}}{q_{1}}}
$$

Now calculate $d m_{2} / d t$ using (3.3) as:

$$
\begin{aligned}
\frac{d}{d t}\left(\frac{a_{2}}{a_{1}}\right) & =\frac{\lambda_{2}}{\lambda_{1}} \frac{d}{d t}\left(\sqrt{\frac{q_{2}}{q_{1}}}\right) \\
& =\frac{\lambda_{2}}{\lambda_{1}} \sqrt{\frac{q_{2}}{q_{1}}} \frac{1}{2} \frac{d}{d t}\left(\log q_{2}-\log q_{1}\right) \\
& =\frac{1}{2} m_{2}\left(\left(a_{3}-a_{1}\right)^{2}-a_{2}^{2}\right)-\left(\left(a_{3}-a_{2}\right)^{2}-a_{1}^{2}\right) \\
& =m_{2}\left(a_{1}-a_{2}\right)\left(a_{1}+a_{2}-a_{3}\right) \\
& =a_{1}^{2} m_{2}\left(1-m_{2}\right)\left(1+m_{2}-m_{3}\right) .
\end{aligned}
$$

The formula for $d m_{3} / d t$ follows analogously. 
Remark 3.2. Solutions to equations (3.6) with initial values in $\mathcal{S}_{m}$ (see (2.3)) exist for all time $t \geq 0$. Since $\mathcal{S}_{m}$ is an invariant set, we need only show that $m_{3}$ does not go to $-\infty$ in finite time. It is easy to see that if $m_{3} \geq 0$, then it will stay so. If $m_{3}<0$, then

$$
\frac{d m_{3}}{d t}=m_{3}\left(1-m_{3}\right)\left(1-m_{2}+m_{3}\right) \geq m_{3}-m_{3}^{3} \geq m_{3},
$$

so $m_{3}(t) \geq m_{3}(0) \mathrm{e}^{t}$. Thus $m_{3}(t)$ cannot go to negative infinity for any $t \geq 0$.

Remark 3.3. It is clear that the Ricci flow equations (3.3) for the $q_{i}$ determine the $a_{i}$ by (3.2), however one might ask if the Ricci flow equations for the $a_{i}$ determine the $q_{i}$. This is, in fact, true, since once the $a_{i}$ are an explicit function of $t$, one can determine the $q_{i}$ by explicitly integrating (3.3), which are now explicit functions of $t$. This was first observed in [33].

\section{Dynamics of the ODEs}

\subsection{Dynamics in $\mathbb{R}^{2}$}

In this section we will look at the qualitative behavior of the dynamical system (3.6). The phase plane for the system of ODEs (3.6) is displayed in figure 3, as computed in Matlab. First, we consider the fixed points. Since the right sides of each equation factor into linear terms, it is easy to see that the fixed points of $\left(m_{2}, m_{3}\right)$ are $(0,0),(0, \pm 1),( \pm 1,0),(1,1)$. Also, it is not hard to see that the following curves are preserved by the flow: (i) $m_{2}=0$, (ii) $m_{3}=0$, (iii) $m_{2}=1$, (iv) $m_{3}=1$ and (v) $m_{2}=m_{3}$.

The Jacobian matrix of the right hand side of system (3.6) at a point $\left(m_{2}, m_{3}\right)$ is

$$
\left(\begin{array}{cc}
2 m_{2} m_{3}-m_{3}-3 m_{2}^{2}+1 & m_{2}\left(m_{2}-1\right) \\
m_{3}\left(m_{3}-1\right) & 2 m_{2} m_{3}-m_{2}-3 m_{3}^{2}+1
\end{array}\right)
$$

and so we see that:

(i) $(0,0)$ is an unstable fixed point.

(ii) $(1,1)$ is a stable fixed point.

(iii) $(0,-1)$ and $(-1,0)$ are saddle points. They have stable manifolds tangent to the lines determined by the eigenvectors $(0,1)$ and $(1,0)$, respectively. They have unstable manifolds tangent to the lines determined by the eigenvectors $(2,1)$ and $(1,2)$. 


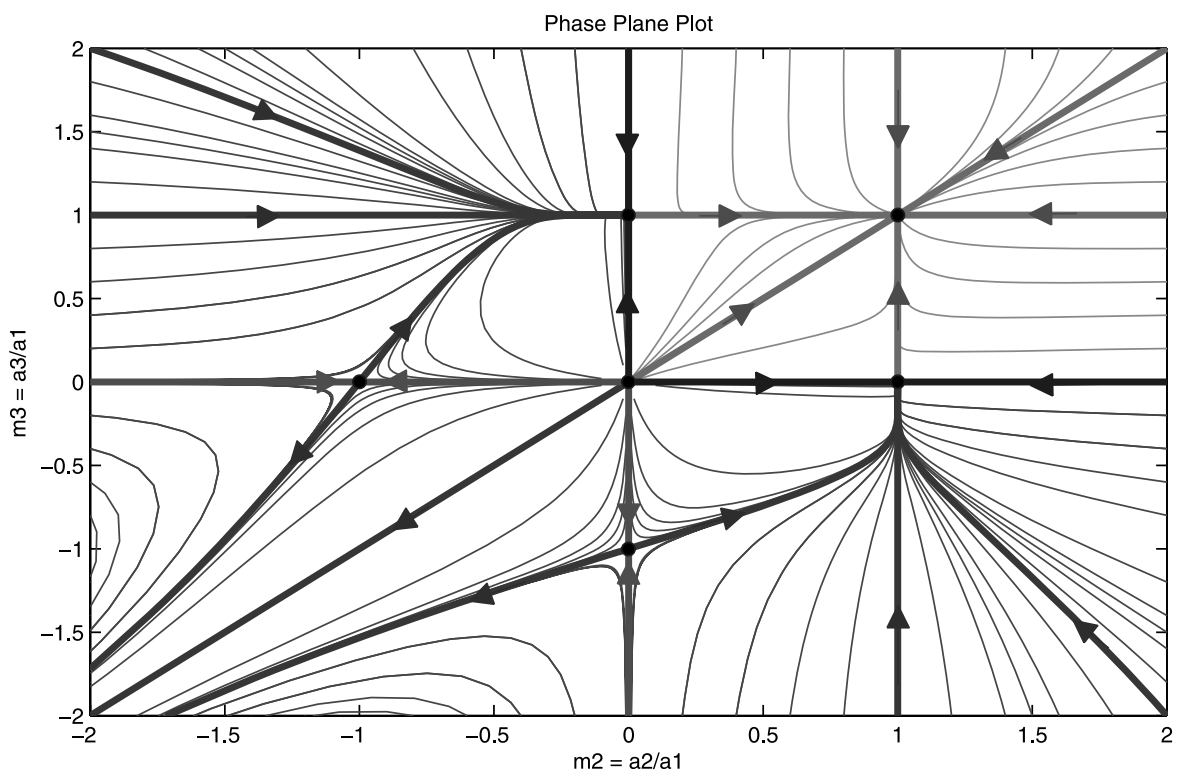

Figure 3: Phase plane for ODEs in equations (3.6).

(iv) $(0,1)$ and $(1,0)$ are degenerate fixed points. They have stable manifolds tangent to the lines determined by the eigenvectors $(0,1)$ and $(1,0)$, respectively. They have a zero eigenvalue corresponding to eigenvectors $(1,0)$ and $(0,1)$; furthermore, one can look in the zero directions by considering the Taylor series of solutions near $s=0$ for the flow along curves $s \rightarrow(s, 1)$ and $s \rightarrow(1, s)$. We see that, for instance, points near $(1,0)$ and below the $x$-axis approach the fixed point, while points above the $x$-axis move away from the fixed point, as seen in Figure 3.

In addition, since $(0,-1)$ and $(-1,0)$ are saddle points (i.e., the linearizations at these points each have two distinct eigenvalues of opposite sign), the Stable Manifold Theorem (see, e.g., [34, Section 2.7]) implies each has a one-dimensional unstable manifold. Although we are unable to calculate the trajectories explicitly, we can, for instance, compute a Taylor approximation of the curve at $(0,-1)$ to be

$$
\begin{aligned}
m_{3}= & -1+\frac{1}{2} m_{2}+\frac{3}{64} m_{2}^{3}+\frac{3}{128} m_{2}^{4}+\frac{9}{512} m_{2}^{5}+\frac{57}{4096} m_{2}^{6} \\
& +\frac{1461}{131072} m_{2}^{7}+O\left(m_{2}^{8}\right) .
\end{aligned}
$$


Furthermore, by the Hartman-Grobman Theorem ([34, Section 2.8]), the trajectories of the differential equation in a neighborhood of $(0,-1)$ are homeomorphic to the trajectories of the linearization around $(0,-1)$, and so this curve contains the only trajectory in the fourth quadrant with $m_{2}<1$ which contains $(0,-1)$.

Remark 4.1. We expect that the Taylor series (4.1) has radius of convergence 1 , since the curve has a vertical tangent at the point $(1,0)$.

\subsection{Dynamics on $\mathcal{M}$}

In this section we prove Theorem A.

Proof of Theorem A. By Theorem 2.2, we can restrict our attention to $\mathcal{S}_{m}$ and consider it up to the equivalence $\sim$ determined by equation (2.4). The discussion in Section 4.1 implies that we have the following fixed points in $\mathcal{S}_{m}$, none of which are equivalent in $\overline{\mathcal{S}}_{m}: p_{1}=(0,0), p_{2}=(0,-1), p_{3}=(1,0)$ and $p_{4}=(1,1)$. It is also clear that for any sequence $\left\{\left(m_{2}^{(i)}, m_{3}^{(i)}\right)\right\}_{i=1}^{\infty}$ with $0 \leq m_{2}^{(i)} \leq 1$ and $\lim _{i \rightarrow \infty} m_{3}^{(i)}=-\infty$, we have

$$
\lim _{i \rightarrow \infty}\left(m_{2}^{(i)}, m_{3}^{(i)}\right)=\infty \sim p_{1}
$$

in $\overline{\mathcal{S}}_{m}$. Define the sets

$$
\begin{aligned}
T_{1,2} & =\left\{\left(m_{2}, m_{3}\right): m_{2}=0,-1<m_{3}<0\right\} \\
& \sim\left\{\left(m_{2}, m_{3}\right): m_{2}=0,-\infty<m_{3}<-1\right\} \\
T_{1,3} & =\left\{\left(m_{2}, m_{3}\right): m_{3}=0,0<m_{2}<1\right\} \\
T_{1,3}^{\prime} & =\left\{\left(m_{2}, m_{3}\right): m_{2}=1,-\infty<m_{2}<0\right\} \\
T_{1,4} & =\left\{\left(m_{2}, m_{3}\right): m_{2}=m_{3}, 0<m_{2}<1\right\} \\
T_{3,4} & =\left\{\left(m_{2}, m_{3}\right): m_{2}=1,0<m_{2}<1\right\} .
\end{aligned}
$$

Notice that these are, in fact, trajectories of the ODEs (3.6), and correspond to the invariant sets described in Section 4.1 .

The special trajectory $T_{2,3}$ is defined as the unstable manifold of the point $p_{2}$ restricted to the set $\left\{\left(m_{2}, m_{3}\right): 0<m_{2}<1\right\}$. As an unstable manifold, it must be invariant. Consider the set $\left\{\left(m_{2}, m_{3}\right): 0<m_{2}<1\right.$ and $\left.m_{3}<0\right\}$. On this set, it follows from (3.6) that if $1-m_{2}+m_{3}=0$ then $\frac{d}{d t}\left(m_{2}, m_{3}\right)$ is in the positive horizontal direction (i.e., $d m_{2} / d t>0$ and 
$\left.d m_{3} / d t=0\right)$. Thus the set

$$
\left\{\left(m_{2}, m_{3}\right): 0<m_{2}<1, m_{3}<0, \text { and } 1-m_{2}+m_{3}<0\right\}
$$

is invariant. Since on this set we have

$$
\frac{d m_{3}}{d m_{2}}=\frac{d m_{3} / d t}{d m_{2} / d t}=\frac{m_{3}\left(1-m_{3}\right)\left(1-m_{2}+m_{3}\right)}{m_{2}\left(1-m_{2}\right)\left(1+m_{2}-m_{3}\right)}>0,
$$

we see that the trajectory $T_{2,3}$ can be written as $m_{3}=f\left(m_{2}\right)$, where $f$ is a continuous, increasing function for $0<m_{2}<1$ such that $f(0)=-1$ and $f(1)=0$.

The remaining sets in the partition of $\mathcal{S}_{m}$ are

$$
\begin{aligned}
& B_{1,4}=\left\{\left(m_{2}, m_{3}\right): 0<m_{2}<1,0<m_{3}<m_{2}\right\}, \\
& B_{1,3}=\left\{\left(m_{2}, m_{3}\right): 0<m_{2}, f\left(m_{2}\right)<m_{3}<0\right\}, \\
& B_{1,3}^{\prime}=\left\{\left(m_{2}, m_{3}\right): 0<m_{2}<1, m_{3}<f\left(m_{2}\right)\right\} .
\end{aligned}
$$

These sets are invariant under the flow of the ODEs since their boundaries are invariant. It remains to show that the sets have the appropriate forward and backwards limit properties; a straightforward analysis of the phase diagram completes the proof.

\section{The geometry of the phase space}

In this section we compare the results of Theorem A with the known geometry of three-dimensional, unimodular metric Lie algebras, thereby proving Theorem B.

Proof of Theorem B. We first look at the fixed points $p_{1}, p_{2}, p_{3}, p_{4}$. Recall that for a three-dimensional, unimodular metric Lie algebra $(\mathfrak{g}, \mathrm{Q})$, we have a basis $\left\{e_{1}, e_{2}, e_{3}\right\}$ as described in Theorem 2.1, and recall that $q_{i}=\mathrm{Q}\left(e_{i}, e_{i}\right)$.

(i) $p_{1}=(0,0)$. This corresponds to the Lie algebra with structure constants $a_{1}=1$ and $a_{2}=a_{3}=0$. By Table 1 we see that this point corresponds to the Heisenberg Lie algebra $\mathfrak{h}(3)$. Up to rescaling, there is only one metric Lie algebra corresponding to $\mathfrak{h}(3)$, and so we see that $p_{1}$ must be that point. This point corresponds to the Ricci soliton on $H(3)$ found by Lauret [24], Baird-Danielo [1] and Lott [29].

(ii) $p_{2}=(0,-1)$. This corresponds to the Lie algebra with structure constants $a_{1}=1, a_{2}=0$, and $a_{3}=-1$. By Table 1 we see that $a_{2}=0$ and 
$a_{3}<0$ determines that this point corresponds to the solvable Lie algebra $\mathfrak{e}(1,1)$. Consulting [12], we see that the left-invariant Riemannian metrics on $E(1,1)$ (referred to as Sol in the reference) have the form

$$
q_{1}\left(\mathrm{e}^{z} d x+\mathrm{e}^{-z} d y\right)^{2}+q_{2} d z^{2}+q_{3}\left(\mathrm{e}^{z} d x-\mathrm{e}^{-z} d y\right)^{2}
$$

and the soliton found by Baird-Danielo [1] and Lott [29] occurs when $q_{1}=q_{3}$. Since at the point $p_{2}$, we have $m_{3}=\sqrt{q_{3} / q_{1}} \lambda_{3}=-1$, we see that $q_{1}=q_{3}$, and so $p_{2}$ corresponds to this soliton metric. Note that among metrics (5.1), the soliton has the form

$$
2 q_{1}\left(\mathrm{e}^{2 z} d x^{2}+\mathrm{e}^{-2 z} d y^{2}\right)+q_{2} d z^{2}
$$

which has the additional symmetry of switching $x$ and $y$. Also note that switching $x$ and $y$ corresponds with switching $q_{1}$ and $q_{3}$, and thus gives precisely the isometry that identifies the metric Lie algebras corresponding to the sets

$$
\left\{\left(m_{2}, m_{3}\right): m_{2}=0,-1<m_{3}<0\right\}
$$

and

$$
\left\{\left(m_{2}, m_{3}\right): m_{2}=0,-\infty<m_{3}<-1\right\},
$$

when using the equivalence relation on $\mathcal{S}_{m}$ from (2.4).

(iii) $p_{3}=(1,0)$. This corresponds to the Lie algebra with structure constants $a_{1}=a_{2}=1$ and $a_{3}=0$. By Table 1 we see that $a_{3}=0$ and $a_{2}>0$ determines that this point corresponds to the solvable Lie algebra $\mathfrak{e}(2)$. Consulting [12], we see that the left-invariant Riemannian metrics on $\widetilde{E(2)}$ (referred to as $\widetilde{\operatorname{Isom}}\left(\mathbb{E}^{2}\right)$ in the reference) have the form

$$
q_{1}(\sin \theta d x+\cos \theta d y)^{2}+q_{2}(\cos \theta d x-\sin \theta d y)^{2}+q_{3} d \theta^{2}
$$

with $q_{1}=q_{2}$ determining the flat metric. We see that $p_{3}$ corresponds to the flat metric. Note that the flat metric is the maximally symmetric metric of the type (5.2).

(iv) $p_{4}=(1,1)$. This corresponds to the Lie algebra $\mathfrak{s u}(2)$ with $a_{1}=a_{2}=$ $a_{3}=1$, implying that $q_{1}=q_{2}=q_{3}$. One easily sees from Theorem 2.1 that this metric has constant sectional curvature, and thus it corresponds to the round metric on the three-sphere. Again, we notice that the round metric is maximally symmetric among all left-invariant metrics on $\mathrm{SU}(2)$. 
We now look at the special trajectories. It is clear that $T_{1,2}$ and $T_{1,3}$ correspond to metrics on $E(1,1)$ and $\widetilde{E(2)}$, respectively, and the explicit metrics shown in (5.1) and (5.2) show the Riemannian submersion structures. In [12], the left-invariant metrics on $\widehat{\mathrm{SL}_{2}(\mathbb{R})}$ are given explicitly, and one sees that on the trajectory $T_{1,3}^{\prime}$ we have $m_{2}=-1$, indicating that $q_{1}=q_{2}$ and that the metrics have the form

$$
q_{1} \frac{1}{y^{2}}\left(d x^{2}+d y^{2}\right)+q_{3}\left(d \theta-\frac{1}{y} d x\right)^{2}
$$

where $(x, y, \theta) \in \mathbb{R} \times \mathbb{R}_{>0} \times \mathbb{R}$. These metrics clearly have the form of Riemannian submersions over the hyperbolic plane.

Now consider the trajectories $T_{1,4}$ and $T_{3,4}$, which we see correspond to metrics on $\mathrm{SU}(2)$. Each element in the basis $\left\{e_{1}, e_{2}, e_{3}\right\}$ exponentiates to a compact group $K$ of rotations in $\mathrm{SU}(2)$. The quotient $\mathrm{SU}(2) / K$ is diffeomorphic to the sphere $S^{2}$, and the map $\pi: \mathrm{SU}(2) \rightarrow \mathrm{SU}(2) / K$ is precisely the Hopf fibration (see, e.g., [3]). Using 9.79 and 9.80 in [3], this can be made into a Riemannian submersion from a left-invariant metric on $\mathrm{SU}(2)$ to a SU(2)-invariant metric on $S^{2}$ with totally geodesic fibers. The Berger spheres are the metrics on $\mathrm{SU}(2)$ which make $\pi$ a Riemannian submersion, where $\mathrm{SU}(2) / K$ is given the round metric on $S^{2}$. The remaining basis elements span the horizontal subspace of the submersion, and thus must have equal length for $\pi$ to be a Riemannian submersion. Thus the submersions are represented only when $m_{2}=1\left(\right.$ so $\left.q_{1}=q_{2}\right)$ or $m_{3}=1\left(\right.$ so $\left.q_{1}=q_{3}\right)$ or $m_{2}=m_{3}\left(\right.$ so $\left.q_{2}=q_{3}\right)$. The round sphere is when $q_{1}=q_{2}=q_{3}$. It is now easy to see that $T_{1,4}$ corresponds to the fibers being larger than the fibers for the round sphere: $q_{2}=q_{3}$ and $m_{2}<1$, so we have $q_{1}>q_{2}=q_{3}$. Similarly, on $T_{3,4}$, we have $q_{1}=q_{2}$ and $m_{3}<1$, so $q_{3}<q_{1}=q_{2}$ and the fibers are smaller than the fibers of the round sphere.

The descriptions of the basins $B_{1,3}, B_{1,3}^{\prime}$, and $B_{1,4}$ follow immediately from Table 1.

Remark 5.1. We could have constructed the Riemannian submersions on $\mathrm{SL}_{2}(\mathbb{R})$ in the same way we constructed the ones for $\mathrm{SU}(2)$ as follows. Instead of considering $\widehat{\mathrm{SL}_{2}(\mathbb{R})}$, consider $\mathrm{PSL}_{2}(\mathbb{R})$, the orientation preserving isometries of $\mathbb{H}^{2}$. There is a compact subgroup $K$ acting on the upper half-plane by the isometries

$$
z \rightarrow \frac{(\cos \theta) z-\sin \theta}{(\sin \theta) z+\cos \theta}
$$


for all angles $\theta$. Note that this is the isotropy group of the point $i$. Again, by 9.79 and 9.80 in [3], there is a Riemannian submersion $\pi: \mathrm{PSL}_{2}(\mathbb{R}) \rightarrow$ $\mathrm{PSL}_{2}(\mathbb{R}) / K$ with totally geodesic fibers, where $\mathrm{PSL}_{2}(\mathbb{R}) / K$ is given the geometry of $\mathbb{H}^{2}$. This submersion may be lifted to the universal cover to get a line bundle over $\mathbb{H}^{2}$.

Remark 5.2. It is an interesting fact that each of the special points and trajectories except for $T_{2,3}$ correspond to metrics with additional symmetry. Generically, the left-invariant metrics have isometry groups of dimension 3 . The point $p_{2}$ is maximally symmetric among left-invariant metrics on $E(1,1)$, containing one extra symmetry, as described in the proof of Theorem B. The points $p_{3}$ and $p_{4}$ have six-dimensional symmetry groups. The trajectories $T_{1,3}^{\prime}, T_{1,4}$, and $T_{3,4}$ have the additional symmetries of reparameterizing the fibers: if the fibration structure is locally trivialized as $(x, s) \in \mathbb{R}^{2} \times S^{1}$, then the map $(x, s) \rightarrow(x, s+\sigma)$ is an isometry. Note that reparameterizing the fiber is different than multiplication by the generator of the fiber in the group. These extra symmetries give $T_{1,3}^{\prime}, T_{1,4}$, and $T_{3,4}$ four-dimensional isometry groups.

\section{Remarks on convergence}

In this section, we compare the convergence results of this paper with other convergence results for Ricci flow on three-dimensional, unimodular Lie groups. Let $(\mathfrak{g}, \mathrm{Q})$ be a metric Lie algebra, and let $(G, g)$ be the corresponding simply connected Lie group with left-invariant Riemannian metric $g$. Any Riemannian manifold $(M, g)$ determines a metric space $\left(M, d_{g}\right)$, where $d_{g}$ is the induced Riemannian distance function. Often it will be relevant to consider quotients $G / \Gamma$ which are manifolds, and in the sequel, we may use $g$ to denote a metric on $G / \Gamma$ as well as on its universal cover $G$. The following are all relevant notions of convergence:

- If the coefficients of the metrics $Q_{t}$ on the Lie algebra, which satisfy the Ricci flow ODEs, converge as $t \rightarrow \infty$, then the corresponding metrics $g(t)$ converge in $C^{k}$ or $C^{\infty}$ as tensors. We call this $C^{k}$ or $C^{\infty}$ convergence.

- The metric spaces $\left(G / \Gamma, d_{g(t)}\right)$ converge uniformly as metric spaces (see [4]).

- The metric spaces $\left(G / \Gamma, d_{g(t)}\right)$ converge in the pointed GromovHausdorff topology (see [13]). 
- The Riemannian groupoids $(G, g(t), \Gamma)$ converge in $C^{k}$ or $C^{\infty}$ as Riemannian groupoids (see $[12,29]$ ).

Before we describe the previous work, let's consider the convergence in the present paper. We have looked at convergence of a system of ODEs for a normalized Ricci flow equation in the space of metric Lie algebras. In particular, the convergence is for the structure constants $a_{1}, a_{2}, a_{3}$, which implies convergence of the connection since the connection is determined by the Lie brackets; in fact, if $D$ is the Riemannian connection and $\left\{e_{1}, e_{2}, e_{3}\right\}$ is an orthonormal frame as in Theorem 2.1, then

$$
\mu_{i}=\frac{1}{2}\left(a_{j}+a_{k}-a_{i}\right)=\left\langle D_{e_{j}} e_{k}, e_{i}\right\rangle
$$

for $\{i, j, k\}=\{1,2,3\}$ (see (2.2)). Convergence in $C^{0}$ of the connections implies convergence in $C^{1}$ of the Riemannian metrics (see, e.g., [8, Chapter 3]). The normalization is not given explicitly, but must be such that none of the Lie bracket coefficients (for an orthonormal frame) become infinite and the Lie algebra does not become abelian. This type of convergence is considered on higher dimensional nilpotent metric Lie algebras in [33].

The earliest works consider either the (forward) Ricci flow equation with no scaling [22] or the normalized Ricci flow equation where the rescaling is based on the scalar curvature [19], and prove $C^{0}$ convergence of the associated left-invariant metrics on the simply connected Lie group. The normalized Ricci flow is helpful for $\mathrm{SU}(2)$, since it prevents the sphere from shrinking to a point in finite time, but otherwise the Ricci flows exist for all time $t$ (for both unnormalized and normalized). Some of these geometries collapse, in the sense that some of the metric coefficients $q_{1}, q_{2}, q_{3}$ go to zero, indicating a compact quotient will have the volume go to zero, as the sectional curvatures go to zero at a rate of $1 / t$. In [7], $C^{0}$ convergence of the backward direction (of normalized Ricci flow) is considered, and it is found that the curvatures may go to infinity and convergence is often in a Gromov-Hausdorff sense to a sub-Riemannian geometry. Similar work is done for the cross curvature flow in $[5,6]$. In each of these cases, compact quotients are considered, and limits are considered collapsing if a fundamental domain has injectivity radius going to zero as time goes to infinity. In many cases, compact quotients collapse with bounded curvature, i.e., the injectivity radius goes to zero while the sectional curvatures stay bounded.

If one is interested in the simply connected Lie groups, which are diffeomorphic to $\mathbb{R}^{3}$ in all cases except $\mathrm{SU}(2)$, the collapsing does not occur. Instead, one can show pointed Gromov-Hausdorff convergence to Euclidean 
space (see [12]). This convergence is sometimes due to the fact that the flow is stretching the geometry, revealing only that the Riemannian manifold is locally Euclidean. To counteract this effect, Lott [29] considers convergence when the metric is rescaled by $1 / t$ to try to prevent the sectional curvatures from decaying to zero. In addition, Lott introduces $C^{k}$ convergence on Riemannian groupoids (see also [12]), which essentially means that the universal cover converges in $C^{k}$ while the actions of the fundamental group change as the metric evolves. In this setting, one finds that the rescaled solutions on the universal covers converge to expanding soliton metrics, getting results similar to those in this paper. It would not be hard to carry out a similar analysis in the backward time direction with the use of [7]. If one is not concerned with a change of topology, the methods of uniform convergence of metric spaces will suffice, as in [4].

We note that in $[12,29]$, only $C^{0}$ convergence of the metrics is shown explicitly, although $C^{\infty}$ convergence of Riemannian groupoids is claimed. It is also shown that the sectional curvatures converge, and so using standard compactness arguments (see, for instance,[11, 18] [8, Chapter 3]), a subsequence of metrics converges in $C^{1}$. Under Ricci flow, Shi's work $([35,36])$ implies that if the curvature is bounded at $t=0$, then all covariant derivatives of curvature are bounded uniformly for $t \geq \delta>0$. Thus, a subsequence of metrics converges in $C^{\infty}$. However, since we are considering rescaling at time $t=0$, Shi's estimates do not directly apply (unless we assume uniform backwards existence), so an additional argument is needed to show that the metrics converge in $C^{k}$ for $k>1$. We would like to have at least convergence in $C^{2}$. The cases in $[12,29]$ can be shown to converge in $C^{k}$ with additional work bounding the covariant derivative. We do not pursue this here, but do present an interesting example below concerning a rescaling that does not produce $C^{\infty}$ convergence.

There is one major difference between the results in this paper and the results in $[12,29]$. Metric Lie algebras of type $\widehat{\mathrm{SL}_{2}(\mathbb{R})}$ converge to flat metrics in our setting, while they converge to $\mathbb{H}^{2} \times \mathbb{R}$ in the setting of $[12,29]$. We note that $\mathbb{H}^{2} \times \mathbb{R}$ cannot be realized as a three-dimensional, unimodular Lie group with a left-invariant metric. We see that, if we rescale the metric to ensure that the sectional curvatures do not go to zero, the Lie algebra coefficients cannot stay bounded: using the computations in [12], we see that

$$
\begin{aligned}
q_{1}, q_{3} & \sim 2 t, \\
q_{2} & \sim E_{1},
\end{aligned}
$$

for some constant $E_{1}>0$, and two sectional curvatures are asymptotically like $1 / t^{2}$ while one is asymptotically like $-1 / t$. The best we can do by 
rescaling is to maintain the negative sectional curvature and let the other two curvatures go to zero. This suggests scaling the metric by $1 / t$. Under this rescaling, using (3.2) we can calculate that

$$
\begin{aligned}
a_{1}, a_{3} & \sim\left(\frac{t}{E_{1}}\right)^{1 / 2}, \\
a_{2} & \sim \frac{1}{2}\left(\frac{E_{1}}{t}\right)^{1 / 2} .
\end{aligned}
$$

Thus we see that this rescaling would not stay in the space of unimodular metric Lie algebras, and we must take a different scaling to stay in this space. A different scaling will cause the sectional curvatures all to go to zero (for instance no scaling at all), revealing Euclidean space.

Metric Lie algebras of the type $\widetilde{E(2)}$ also converge to a flat metric, so one might ask if rescaling by the maximum curvature can create a non-flat limit. We will use the notation of [12]. Note that

$$
a_{2}-a_{1}=\frac{q_{2}-q_{1}}{\sqrt{q_{1} q_{2} q_{3}}}
$$

and recall that in this case, Ricci flow has

$$
\begin{aligned}
q_{1}, q_{2} & \sim E_{1} \\
q_{3} & \sim E_{3} \\
q_{1}-q_{2} & \sim E_{4} \mathrm{e}^{-E_{3} t},
\end{aligned}
$$

for constants $E_{1}, E_{2}, E_{3}, E_{4}$, and sectional curvatures are all proportional to $\mathrm{e}^{-E_{3} t}$. If we rescale, replacing $q_{i}$ with $\mathrm{e}^{-E_{3} t} q_{i}$, we see that

$$
\begin{aligned}
a_{2}-a_{1} & \sim \frac{E_{4} \mathrm{e}^{-2 E_{3} t}}{E_{5} \mathrm{e}^{-(3 / 2) E_{3} t}}=E_{6} \mathrm{e}^{-(1 / 2) E_{3} t}, \\
a_{1}, a_{2} & \sim E_{7} \mathrm{e}^{(1 / 2) E_{3} t} \\
a_{3} & =0 .
\end{aligned}
$$

Thus for the rescaled solution,

$$
\begin{aligned}
& \mu_{1} \sim \frac{1}{2} E_{6} \mathrm{e}^{-(1 / 2) E_{3} t} \\
& \mu_{2} \sim-\frac{1}{2} E_{6} \mathrm{e}^{-(1 / 2) E_{3} t} \\
& \mu_{3} \sim E_{7} \mathrm{e}^{(1 / 2) E_{3} t}
\end{aligned}
$$


and thus, using Proposition 2.1,

$$
\begin{aligned}
|D \mathrm{Rc}|^{2} \sim & 8\left(\left(E_{7} \mathrm{e}^{(1 / 2) E_{3} t}\right)^{2}\left(\frac{1}{2} E_{6} \mathrm{e}^{-(1 / 2) E_{3} t}\right)^{4}+\left(E_{6} \mathrm{e}^{-(1 / 2) E_{3} t}\right)^{2}\left(E_{7} \mathrm{e}^{(1 / 2) E_{3} t}\right)^{4}\right. \\
& \left.+\left(E_{7} \mathrm{e}^{(1 / 2) E_{3} t}\right)^{2}\left(\frac{1}{2} E_{6} \mathrm{e}^{-(1 / 2) E_{3} t}\right)^{4}\right) \\
& \sim 8 E_{6}^{2} E_{7}^{4} \mathrm{e}^{E_{3} t}
\end{aligned}
$$

and so under this rescaling, $|D \mathrm{Rc}|^{2} \rightarrow \infty$ as $t \rightarrow \infty$. Since $|D \mathrm{Rc}|^{2}$ is not bounded, we cannot use Arzela-Ascoli-type compactness theorems to get convergence of the curvatures (see [11] or [8]) and the curvatures may not converge. Thus this kind of rescaling does not necessarily result in $C^{2}$ and definitely not $C^{3}$ convergence. Thus there is no natural rescaling which results in a non-flat limit.

We can also investigate what happens in the backwards time limit and compare to the results of [7]. The results are slightly different, since once again the rescaling in [7] is chosen in a particular way, while we have chosen a different rescaling. A comparison can be made in a straightforward way, which we leave to the reader. However, we would like to point out the particularly interesting case of the backwards limit of the trajectory $T_{3,4}$, which appears to converge, in our setting, to the point $p_{3}$ (see figure 3), which represents the flat metric on $\widetilde{E(2)}$ (see Theorem B). As in the case

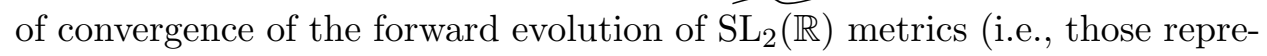
sented by $B_{1,3}, B_{1,3}^{\prime}, T_{2,3}$, and $T_{1,3}^{\prime}$ as in Theorem $\mathrm{A}$ ), the fact that we see convergence to the flat metric indicates that our rescaling may be revealing only the infinitesimal Euclidean character of the limit. In fact, as we saw in Section 5, the trajectory $T_{3,4}$ corresponds to Berger metrics which are Riemannian submersions over the round two-sphere. As we follow the trajectory backwards, the fibers shrink and so we expect convergence to the two-sphere. The formalism in $[12,29]$ together with the calculations in [7] allow one to make this convergence precise in the sense of Riemannian groupoids (and, in particular, pointed Gromov-Hausdorff).

Finally, we note that the stability analysis of Ricci solitons differs from that in [14]. In [14], only compactly supported variations are considered, and it was found, for instance, that the soliton metric on $H(3)$ is linearly stable. We found that in the space of three-dimensional, nonabelian, unimodular metric Lie algebras up to scaling, the soliton metric on $H(3)$ is unstable (it corresponds to a repulsive fixed point). These two results are not contradictory, since the variations we consider are certainly not compactly supported. 


\section{Appendix A. Three-dimensional, unimodular metric Lie algebras}

In this appendix, we prove a characterization of the space of three-dimensional, nonabelian, unimodular metric Lie algebras, considered up to isometry and scaling. Under the correspondence of Lemma 2.1, three-dimensional, unimodular metric Lie algebras correspond to vectors $\left(a_{1}, a_{2}, a_{3}\right) \in \mathbb{R}^{3}$ by formula (2.1). To account for equivalence under scaling, we consider twodimensional real projective space $\mathbb{R P}^{2} \cong\left(\mathbb{R}^{3} \backslash\{(0,0,0)\}\right) / \mathbb{R}^{\times}$, and denote the image of $(x, y, z) \in \mathbb{R}^{3} \backslash\{(0,0,0)\}$ in $\mathbb{R P}^{2}$ under the quotient map as $(x: y: z)$. Permuting the components induces an isometry of the metric Lie algebra, and so we define the action of $\sigma \in S_{3}$, the group of permutations of three elements, on $\mathbb{R P}^{2}$ by $\sigma\left(a_{1}: a_{2}: a_{3}\right)=\left(a_{\sigma(1)}: a_{\sigma(2)}: a_{\sigma(3)}\right)$. Notice that the actions of $S_{3}$ and $\mathbb{R}^{\times}$on $\mathbb{R}^{3} \backslash\{(0,0,0)\}$ are commutative with respect to each other, and thus

$$
\mathbb{R P}^{2} / S_{3} \cong\left(\mathbb{R}^{3} \backslash\{(0,0,0)\}\right) /\left(\mathbb{R}^{\times} \times S_{3}\right)
$$

We denote the image of $(x, y, z) \in \mathbb{R}^{3} \backslash\{(0,0,0)\}$ in $\mathbb{R P}^{2} / S_{3}$ as $[x: y: z]$, where the use of square brackets instead of round brackets indicates the further equivalence using the $S_{3}$ action. We will now introduce a fundamental domain for $\mathbb{R P}^{2} / S_{3}$. Recall the sets $\mathcal{S}_{m}$ and $\overline{\mathcal{S}}_{m}$ defined in (2.3) and (2.5) and the equivalence relation $\sim$ determined by (2.4) and (2.6).

Proposition A.1. The map

$$
\tilde{\Phi}: \overline{\mathcal{S}}_{m} \rightarrow \mathbb{R P}^{2} / S_{3}
$$

defined by

$$
\begin{aligned}
\tilde{\Phi}\left(m_{2}, m_{3}\right) & =\left[1: m_{2}: m_{3}\right] \\
\tilde{\Phi}(\infty) & =[1: 0: 0]
\end{aligned}
$$

is surjective. Moreover, $\tilde{\Phi}$ induces a homeomorphism

$$
\Phi: \overline{\mathcal{S}}_{m} / \sim \longrightarrow \mathbb{R P}^{2} / S_{3}
$$

Proof. Consider a point $[x: y: z] \in \mathbb{R P}^{2} / S_{3}$. We can use multiplication by -1 to ensure that at least one entry is positive and another is nonnegative. Then we can use the permutations to ensure that $z \leq y \leq x, y \geq 0$, and 
$x>0$. In this case we have that

$$
[x: y: z]=\left[1: \frac{y}{x}: \frac{z}{x}\right]
$$

with

$$
0 \leq \frac{y}{x} \leq 1
$$

and

$$
\frac{z}{x} \leq \frac{y}{x}
$$

which proves the first statement.

To see that $\Phi$ is well-defined, we must check that $\tilde{\Phi}\left(0, m_{3}\right)=\tilde{\Phi}\left(0,1 / m_{3}\right)$ for $m_{3} \neq 0$. We see that

$$
\left[1: 0: m_{3}\right]=\left[\frac{1}{m_{3}}: 0: 1\right]=\left[1: 0: \frac{1}{m_{3}}\right]
$$

To see $\Phi$ is a bijection, we note that if

$$
\tilde{\Phi}\left(m_{2}, m_{3}\right)=\tilde{\Phi}\left(m_{2}^{\prime}, m_{3}^{\prime}\right)
$$

then

$$
\left[1: m_{2}: m_{3}\right]=\left[1: m_{2}^{\prime}: m_{3}^{\prime}\right]
$$

with

$$
\begin{aligned}
& 0 \leq m_{2} \leq 1, \quad m_{3} \leq m_{2} \\
& 0 \leq m_{2}^{\prime} \leq 1, \quad m_{3}^{\prime} \leq m_{2}^{\prime}
\end{aligned}
$$

Thus $\left(1, m_{2}, m_{3}\right)$ is equal to one of the following, where $r \neq 0: r\left(1, m_{2}^{\prime}, m_{3}^{\prime}\right)$, $r\left(1, m_{3}^{\prime}, m_{2}^{\prime}\right), r\left(m_{2}^{\prime}, 1, m_{3}^{\prime}\right), r\left(m_{3}^{\prime}, 1, m_{2}^{\prime}\right), r\left(m_{3}^{\prime}, m_{2}^{\prime}, 1\right)$ or $r\left(m_{2}^{\prime}, m_{3}^{\prime}, 1\right)$. Вy checking each of the cases, one can verify that $\Phi$ is a bijection.

To see the continuity, we note that for any sequence $\left(m_{2}^{(i)}, m_{3}^{(i)}\right)$ satisfying $0 \leq m_{2}^{(i)} \leq 1$ and $\lim _{i \rightarrow \infty} m_{3}^{(i)}=-\infty$, we have

$$
\begin{aligned}
\lim _{i \rightarrow \infty} \tilde{\Phi}\left(m_{2}^{(i)}, m_{3}^{(i)}\right) & =\lim _{i \rightarrow \infty}\left[1: m_{2}^{(i)}: m_{3}^{(i)}\right] \\
& =\lim _{i \rightarrow \infty}\left[1: \frac{m_{2}^{(i)}}{m_{3}^{(i)}}: \frac{1}{m_{3}^{(i)}}\right] \\
& =[1: 0: 0] .
\end{aligned}
$$


Certainly there is a map

$$
\Psi: \mathbb{R P}^{2} / S_{3} \rightarrow \mathcal{M}
$$

induced by the map $\tilde{\Psi}$ defined in Lemma 2.1. We wish to show that this map is bijection. It is immediate that the map is surjective, so we need only show that it is injective. The main ideas for the proof are in the paper of Lastaria [23], which constructs families of nonisometric metric Lie algebras with the same Ricci tensors.

We will need two invariants for $\mathcal{M}$. It is easy to see that the following quantities are invariants of $\mathcal{M}$ if $|R c| \neq 0$ (and the case $|R c|=0$ is distinguished from these cases as well):

$$
\begin{array}{r}
\frac{\left(\lambda_{1}[\mathrm{Rc}], \lambda_{2}[\mathrm{Rc}], \lambda_{3}[\mathrm{Rc}]\right)}{|\mathrm{Rc}|} \in S^{2} \\
\frac{|D \mathrm{Rc}|^{2}}{|\mathrm{Rc}|^{3}} \in \mathbb{R},
\end{array}
$$

where $\lambda_{1}[\mathrm{Rc}] \leq \lambda_{2}[\mathrm{Rc}] \leq \lambda_{3}[\mathrm{Rc}]$ are the eigenvalues of the Ricci operator put into ascending order. Notice that $|\mathrm{Rc}|=0$ only if $\left(m_{2}, m_{3}\right)=(1,0)$, and so the corresponding metric Lie algebra is not isometric to any metric Lie algebra induced by another element of $\overline{\mathcal{S}}_{m} / \sim$.

We will consider the map

$$
\tilde{E}=E \circ \Psi \circ \tilde{\Phi}: \mathcal{S}_{m} \backslash\{(1,0)\} \rightarrow S^{2} \times \mathbb{R}
$$

where

$$
E: \mathcal{M} \rightarrow S^{2} \times \mathbb{R}
$$

gives the two invariants above. Recall that the Ricci eigenvalues are

$$
\left(2 \mu_{2} \mu_{3}, 2 \mu_{1} \mu_{3}, 2 \mu_{1} \mu_{2}\right)
$$

where $\mu_{1}, \mu_{2}, \mu_{3}$ are as in Theorem 2.1. We want to express these in terms of $m_{2}$ and $m_{3}$, so we introduce the following functions which are closely related to the $\mu_{i}$ :

$$
\begin{aligned}
& \nu_{1}=m_{2}+m_{3}-1, \\
& \nu_{2}=1+m_{3}-m_{2}, \\
& \nu_{3}=1+m_{2}-m_{3} .
\end{aligned}
$$


Recall that on $\mathcal{S}_{m}$ we have $m_{3} \leq m_{2}$, and so $\nu_{3} \geq 1$. Also, since $0 \leq m_{2} \leq 1$, we have

$$
-\nu_{3} \leq \nu_{1} \leq \nu_{2}
$$

We define the partition

$$
P=\left\{S_{0}, S_{1}, S_{2}, S_{++}, S_{-+}, S_{++}\right\}
$$

of $\mathcal{S}_{m}$ by looking at the signs of $\nu_{1}$ and $\nu_{2}$ :

$$
\begin{aligned}
S_{0} & =\{(1,0)\} \\
S_{1} & =\left\{\left(m_{2}, m_{3}\right) \in \mathcal{S}_{m}: \nu_{1}=0<\nu_{2}\right\} \\
S_{2} & =\left\{\left(m_{2}, m_{3}\right) \in \mathcal{S}_{m}: \nu_{1}<\nu_{2}=0\right\} \\
S_{++} & =\left\{\left(m_{2}, m_{3}\right) \in \mathcal{S}_{m}: 0<\nu_{1} \leq \nu_{2}\right\} \\
S_{-+} & =\left\{\left(m_{2}, m_{3}\right) \in \mathcal{S}_{m}: \nu_{1}<0<\nu_{2}\right\} \\
S_{--} & =\left\{\left(m_{2}, m_{3}\right) \in \mathcal{S}_{m}: \nu_{1} \leq \nu_{2}<0\right\} .
\end{aligned}
$$

Using Theorem 2.1 and Proposition 2.1 we can write $\tilde{E}$, which is defined on $\mathcal{S}_{m} \backslash S_{0}$, explicitly as follows:

$$
\tilde{E}\left(m_{2}, m_{3}\right)= \begin{cases}\left((0,0,1), \frac{\left(\nu_{2}^{2}+\nu_{3}^{2}\right)}{\nu_{2} \nu_{3}}\right) & \text { if }\left(m_{2}, m_{3}\right) \in S_{1} \\ \left(\frac{\left(\nu_{1} \nu_{2}, \nu_{1} \nu_{3}, \nu_{2} \nu_{3}\right)}{|\nu \nu|}, *\right) & \text { if }\left(m_{2}, m_{3}\right) \in S_{++} \\ \left(\frac{\left(\nu_{1} \nu_{3}, \nu_{1} \nu_{2}, \nu_{2} \nu_{3}\right)}{|\nu \nu|}, *\right) & \text { if } \left.\left(m_{2}, m_{3}\right) \in S_{2}, m_{3}\right) \in S_{-+} \\ \left(\frac{\nu_{1} \nu_{3}, \nu_{2} \nu_{3}, \nu_{1} \nu_{2}}{|\nu \nu|}, *\right) & \text { if }\left(m_{2}, m_{3}\right) \in S_{--},\end{cases}
$$

where

$$
*=\frac{\left(\nu_{1}-\nu_{3}\right)^{2} \nu_{2}^{4}+\left(\nu_{1}-\nu_{2}\right)^{2} \nu_{3}^{4}+\left(\nu_{2}-\nu_{3}\right)^{2} \nu_{1}^{4}}{|\nu \nu|^{3}}
$$

and

$$
|\nu \nu|=\sqrt{\nu_{1}^{2} \nu_{2}^{2}+\nu_{1}^{2} \nu_{3}^{2}+\nu_{2}^{2} \nu_{3}^{2}} .
$$


Now we will show that $\tilde{E}$ is injective. First we will show that no two points in different partitions in $P$ correspond to equivalent metric Lie algebras (equivalent up to isometry and scaling). Then we will show that within each partition, no two points correspond to equivalent metric Lie algebras.

Proposition A.2. If $\left(m_{2}, m_{3}\right)$ and $\left(m_{2}^{\prime}, m_{3}^{\prime}\right)$ are in different sets from the partition $P($ see $(A .3))$, then $\tilde{E}\left(m_{2}, m_{3}\right)=\tilde{E}\left(m_{2}^{\prime}, m_{3}^{\prime}\right)$ implies that

- $\left(m_{2}, m_{3}\right)=\left(0, m_{3}\right) \in S_{-+}$and $\left(m_{2}^{\prime}, m_{3}^{\prime}\right)=\left(0,1 / m_{3}\right) \in S_{--}$or

- $\left(m_{2}, m_{3}\right)=\left(0, m_{3}\right) \in S_{--}$and $\left(m_{2}^{\prime}, m_{3}^{\prime}\right)=\left(0,1 / m_{3}\right) \in S_{-+}$.

Proof. Since $\tilde{E}$ restricted to $S_{++} \cup S_{-+} \cup S_{--}$does not have any zeroes in the first component, then certainly we have $\tilde{E}\left(m_{2}, m_{3}\right) \neq \tilde{E}\left(m_{2}^{\prime}, m_{3}^{\prime}\right)$ if $\left(m_{2}, m_{3}\right)$ or $\left(m_{2}^{\prime}, m_{3}^{\prime}\right)$ is in $S_{1}$ or $S_{2}$. Furthermore, since the first component has all positive entries if $\left(m_{2}, m_{3}\right) \in S_{++}$, it is distinguished from $S_{-+}$and $S_{--}$, both of which have two negative entries. This shows that $\tilde{E}\left(m_{2}, m_{3}\right) \neq$ $\tilde{E}\left(m_{2}^{\prime}, m_{3}^{\prime}\right)$ for all cases except if $\left(m_{2}, m_{3}\right)$ or $\left(m_{2}^{\prime}, m_{3}^{\prime}\right)$ in $S_{-+}$and the other in $S_{--}$. Say $\left(m_{2}, m_{3}\right) \in S_{-+}$and $\left(m_{2}^{\prime}, m_{3}^{\prime}\right) \in S_{--}$and $\tilde{E}\left(m_{2}, m_{3}\right)=$ $\tilde{E}\left(m_{2}^{\prime}, m_{3}^{\prime}\right)$. Thus we have that

$$
\frac{\left(\nu_{1} \nu_{3}, \nu_{1} \nu_{2}, \nu_{2} \nu_{3}\right)}{|\nu \nu|}=\frac{\left(\nu_{1}^{\prime} \nu_{3}^{\prime}, \nu_{2}^{\prime} \nu_{3}^{\prime}, \nu_{1}^{\prime} \nu_{2}^{\prime}\right)}{\left|\nu^{\prime} \nu^{\prime}\right|}
$$

with

$$
\begin{aligned}
\nu_{1} & <0<\nu_{2} \\
\nu_{1}^{\prime} & \leq \nu_{2}^{\prime}<0 \\
0 & <\nu_{3}, \nu_{3}^{\prime} .
\end{aligned}
$$

That is, there exists $c>0$ such that

$$
\frac{\nu_{1} \nu_{3}}{\nu_{1}^{\prime} \nu_{3}^{\prime}}=\frac{\nu_{1} \nu_{2}}{\nu_{2}^{\prime} \nu_{3}^{\prime}}=\frac{\nu_{2} \nu_{3}}{\nu_{1}^{\prime} \nu_{2}^{\prime}}=c,
$$

which implies

$$
\nu_{1} \nu_{2}^{2} \nu_{3}=c^{2} \nu_{1}^{\prime}\left(\nu_{2}^{\prime}\right)^{2} \nu_{3}^{\prime}
$$

Using (A.5), we find that

$$
\nu_{2}^{2}=c\left(\nu_{2}^{\prime}\right)^{2},
$$

and, using (A.4) and (A.5) again, we arrive at

$$
\nu_{2}=-\sqrt{c} \nu_{2}^{\prime}, \quad \nu_{3}=-\sqrt{c} \nu_{1}^{\prime}, \quad \nu_{1}=-\sqrt{c} \nu_{3}^{\prime} .
$$


It is a consequence of (A.2) that

$$
-\nu_{3} \leq \nu_{1}=-\sqrt{c} \nu_{3}^{\prime} \leq \sqrt{c} \nu_{1}^{\prime}=-\nu_{3}
$$

So

$$
\nu_{1}=-\nu_{3} \text { and } \nu_{1}^{\prime}=-\nu_{3}^{\prime}
$$

Thus, using (A.1),

$$
\begin{aligned}
& m_{2}+m_{3}-1=-1-m_{2}+m_{3} \\
& m_{2}^{\prime}+m_{3}^{\prime}-1=-1-m_{2}^{\prime}+m_{3}^{\prime}
\end{aligned}
$$

or $m_{2}=m_{2}^{\prime}=0$. Again by (A.1), we have the following:

$$
\begin{aligned}
\nu_{1} & =-\nu_{3}=m_{3}-1 \\
\nu_{2} & =1+m_{3} \\
\nu_{1}^{\prime} & =-\nu_{3}^{\prime}=m_{3}^{\prime}-1 \\
\nu_{2}^{\prime} & =1+m_{3}^{\prime} .
\end{aligned}
$$

Inserting these into (A.5) gives

$$
\frac{\left(m_{3}-1\right)^{2}}{\left(m_{3}^{\prime}-1\right)^{2}}=\frac{m_{3}^{2}-1}{1-\left(m_{3}^{\prime}\right)^{2}}
$$

which implies that

$$
\left(m_{3}-1\right)^{2}\left(1-\left(m_{3}^{\prime}\right)^{2}\right)-\left(m_{3}^{2}-1\right)\left(m_{3}^{\prime}-1\right)^{2}=0
$$

or

$$
\left(m_{3}-1\right)\left(m_{3}^{\prime}-1\right)\left(m_{3} m_{3}^{\prime}-1\right)=0
$$

Since neither $m_{3}$ nor $m_{3}^{\prime}$ can equal one, we must have $m_{3} m_{3}^{\prime}=1$.

Proposition A.3. $\tilde{E}$ is injective on $S_{1}$. 
Proof. On $S_{1}$, we have

$$
0=\nu_{1}=m_{2}+m_{3}-1
$$

which implies that

$$
m_{3}=1-m_{2} \text {. }
$$

Notice that this implies that

$$
1-m_{2}=m_{3} \leq m_{2},
$$

or

$$
\frac{1}{2} \leq m_{2}<1
$$

(using that $m_{2}=1$ implies $m_{3}=0$ ). Now we can write $\nu_{2}$ and $\nu_{3}$ in terms of $m_{2}$ :

$$
\begin{aligned}
\nu_{2} & =1+m_{3}-m_{2}=2-2 m_{2}, \\
\nu_{3} & =1+m_{2}-m_{3}=2 m_{2},
\end{aligned}
$$

So

$$
\frac{\left(\nu_{2}^{2}+\nu_{3}^{2}\right)}{\nu_{2} \nu_{3}}=\frac{\left(1-m_{2}\right)^{2}+m_{2}^{2}}{\left(1-m_{2}\right) m_{2}} .
$$

This function is one-to-one on $1 / 2 \leq m_{2}<1$. Thus $\tilde{E}$ is injective.

Proposition A.4. $\tilde{E}$ is injective on $S_{2}$.

Proof. On $S_{2}$, we have

$$
0=\nu_{2}=1+m_{3}-m_{2}
$$

which implies that

$$
m_{3}=m_{2}-1 \text {. }
$$

Now we can write $\nu_{1}$ and $\nu_{3}$ in terms of $m_{2}$

$$
\begin{aligned}
& \nu_{1}=m_{2}+m_{3}-1=2 m_{2}-2 \\
& \nu_{3}=1+m_{2}-m_{3}=2
\end{aligned}
$$

so

$$
\frac{\left(\nu_{1}^{2}+\nu_{3}^{2}\right)}{\left|\nu_{1} \nu_{3}\right|}=\frac{\left(m_{2}-1\right)^{2}+1}{1-m_{2}} .
$$

This function is strictly increasing for $0<m_{2}<1$. The result follows. 
Note that if $U \subset S^{2} \cap\{(x, y, z): z>0\}$ then the map $\phi: U \rightarrow \mathbb{R}^{2}$ defined by $\phi(x, y, z)=\left(\frac{x}{z}, \frac{y}{z}\right)$ is one-to-one. We will use this to prove that $\tilde{E}$ is injective on the sets $S_{++}, S_{-+}, S_{--}$.

Proposition A.5. $\tilde{E}$ is injective on each of the sets $S_{++}, S_{-+}$, and $S_{--}$.

Proof. Let $\pi_{1}$ be the projection onto the first component and let $\bar{E}=\phi \circ$ $\pi_{1} \circ \tilde{E}$. On $S_{++}, \bar{E}$ is

$$
\bar{E}\left(m_{2}, m_{3}\right)=\left(\frac{\nu_{1}}{\nu_{3}}, \frac{\nu_{1}}{\nu_{2}}\right) .
$$

We compute the Jacobian determinant:

$$
\operatorname{det}\left(\begin{array}{cc}
\frac{2\left(1-m_{3}\right)}{\nu_{3}^{2}} & \frac{2 m_{2}}{\nu_{3}^{2}} \\
\frac{2 m_{3}}{\nu_{2}^{2}} & \frac{2\left(1-m_{2}\right)}{\nu_{2}^{2}}
\end{array}\right)=\frac{-4 \nu_{1}}{\nu_{2}^{2} \nu_{3}^{2}}<0
$$

since $\nu_{1}>0$ on $S_{++}$. On $S_{-+}, \bar{E}$ is

$$
\bar{E}\left(m_{2}, m_{3}\right)=\left(\frac{\nu_{1}}{\nu_{2}}, \frac{\nu_{1}}{\nu_{3}}\right) .
$$

Its Jacobian determinant is

$$
\operatorname{det}\left(\begin{array}{cc}
\frac{2 m_{3}}{\nu_{2}^{2}} & \frac{2\left(1-m_{2}\right)}{\nu_{2}^{2}} \\
\frac{2\left(1-m_{3}\right)}{\nu_{3}^{2}} & \frac{2 m_{2}}{\nu_{3}^{2}}
\end{array}\right)=\frac{4 \nu_{1}}{\nu_{2}^{2} \nu_{3}^{2}}<0
$$

since $\nu_{1}<0$ on $S_{-+}$. On $S_{--}, \bar{E}$ is

$$
\bar{E}\left(m_{2}, m_{3}\right)=\left(\frac{\nu_{3}}{\nu_{2}}, \frac{\nu_{3}}{\nu_{1}}\right) .
$$

Its Jacobian determinant is

$$
\operatorname{det}\left(\begin{array}{cc}
\frac{2}{\nu_{2}^{2}} & \frac{-2}{\nu_{2}^{2}} \\
\frac{2\left(m_{3}-1\right)}{\nu_{1}^{2}} & \frac{-2 m_{2}}{\nu_{1}^{2}}
\end{array}\right)=\frac{-4 \nu_{3}}{\nu_{1}^{2} \nu_{2}^{2}}>0 .
$$

In each case we see that $\bar{E}$ is injective, and thus so is $\tilde{E}$. 
As a consequence of all of these propositions, we can conclude that $\tilde{E}$ is injective, implying the following theorem, which implies Theorem 2.2. Note that we have not yet defined a topology on $\mathcal{M}$; it will be defined to allow for the theorem.

Theorem A.1. The following spaces are homeomorphic:

(i) $\mathcal{M}$

(ii) $\mathbb{R P}^{2} / S_{3}$

(iii) $\overline{\mathcal{S}}_{m} / \sim$

Proof. We have just shown that $\Psi$ gives a bijection between $\mathbb{R P}^{2} / S_{3}$ and $\mathcal{M}$, and we can give $\mathcal{M}$ a topology which makes $\Psi$ a homeomorphism. Proposition A.1 shows that $\Phi$ is a homeomorphism between $\overline{\mathcal{S}}_{m} / \sim$ and $\mathbb{R P}^{2} / S_{3}$.

\section{Acknowledgments}

DG partially supported by NSF grant DMS 0748283. TLP partially supported by a WeLead grant from NSF Advance grant RRES31.

\section{References}

[1] P. Baird and L. Danielo, Three-dimensional Ricci solitons which project to surfaces, J. Reine Angew. Math. 608 (2007), 65-91.

[2] F. Bourliot, J. Estes, P.M. Petropoulos and P. Spindel, Gravitational instantons, self-duality and geometric flows, Phys. Rev. D81 (2010), 104001.

[3] A.L. Besse, Einstein manifolds, Ergebnisse der Mathematik und ihrer Grenzgebiete (3) [Results in Mathematics and Related Areas (3)] 10, Springer-Verlag, Berlin, 1987, xii+510.

[4] X. Cao, J. Guckenheimer and L. Saloff-Coste, The backward behavior of the Ricci and cross curvature flows on $S L(2, R)$, Comm. Anal. Geom. 17(4) (2009), 777-796.

[5] X. Cao, Y. Ni and L. Saloff-Coste, Cross curvature flow on locally homogenous three-manifolds, I. Pacific J. Math. 236(2) (2008), 263-281.

[6] X. Cao and L. Saloff-Coste, Cross curvature flow on locally homogeneous three-manifolds (II), Asian J. Math. 13(4) (2009), 421-458. 
[7] X. Cao and L. Saloff-Coste, Backward Ricci flow on locally homogeneous 3-manifolds, Comm. Anal. Geom. 17(2) (2009), 305-325.

[8] B. Chow, S.-C. Chu, D. Glickenstein, C. Guenther, J. Isenberg, T. Ivey, D. Knopf, P. Lu, F. Luo and L. Ni, The Ricci flow: techniques and applications. Part I. Geometric aspects, Mathematical Surveys and Monographs, 135, American Mathematical Society, Providence, RI, 2007, $\mathrm{xxiv}+536$.

[9] G.F.R. Ellis, S.T.C. Siklos and J. Wainwright, Geometry of cosmological models, Dynamical Systems in cosmology, Cambridge University Press, Cambridge, UK, 1997, 11-50.

[10] G.F.R. Ellis, C. Uggla and J. Wainwright, Qualitative analysis of Bianchi cosmologies, Dynamical Systems in Cosmology, Cambridge University Press, Cambridge, UK, 1997, 107-122.

[11] D. Glickenstein, Precompactness of solutions to the Ricci flow in the absence of injectivity radius estimates, Geom. Topol. 7 (2003), 487-510 (electronic).

[12] D. Glickenstein, Riemannian groupoids and solitons for threedimensional homogeneous Ricci and cross-curvature flows, Int. Math. Res. Not. 12 (2008), Art. ID rnn034, 49.

[13] M. Gromov, Metric structures for Riemannian and non-Riemannian spaces. Based on the 1981 French original. With appendices by M. Katz, P. Pansu and S. Semmes. Translated from the French by Sean Michael Bates. Reprint of the 2001 English edition. Modern Birkhäuser Classics. Birkhäuser Boston, Inc., Boston, MA, 2007, $\mathrm{xx}+585$.

[14] C. Guenther, J. Isenberg and D. Knopf, Linear stability of homogeneous Ricci solitons, Int. Math. Res. Not. (2006), Art. ID 96253, 30.

[15] G. Guzhvina, Ricci flow on almost flat manifolds, Differential Geometry and its Applications, World Science Publisher, Hackensack, NJ, 2008, $133-146$.

[16] R.S. Hamilton, Three-manifolds with positive Ricci curvature, J. Differential Geom. 17(2) (1982), 255-306.

[17] R.S. Hamilton, The formation of singularities in the Ricci flow. Surveys in differential geometry, II, Cambridge, MA, 1993, Int. Press, Cambridge, MA, 1995, 7-136. 
[18] R.S. Hamilton, A compactness property for solutions of the Ricci flow, Amer. J. Math. 117(3) (1995), 545-572.

[19] J. Isenberg and M. Jackson, Ricci flow of locally homogeneous geometries on closed manifolds, J. Differential Geom. 35(3) (1992), 723-741.

[20] J. Isenberg, M. Jackson and P. Lu, Ricci flow on locally homogeneous closed 4-manifolds, Comm. Anal. Geom. 14(2) (2006), 345-386.

[21] G.R. Jensen, The scalar curvature of left-invariant Riemannian metrics, Indiana Univ. Math. J. 20 (1970/1971), 1125-1144.

[22] D. Knopf and K. McLeod, Quasi-convergence of model geometries under the Ricci flow, Comm. Anal. Geom. 9(4) (2001), 879-919.

[23] F.G. Lastaria, Homogeneous metrics with the same curvature, Simon Stevin 65(3-4) (1991), 267-281.

[24] J. Lauret, Ricci soliton homogeneous nilmanifolds, Math. Ann. 319(4) (2001), 715-733.

[25] J. Lauret, Degenerations of Lie algebras and geometry of Lie groups, Differential Geom. Appl. 18(2) (2003), 177-194.

[26] J. Lauret, Einstein solvmanifolds and nilsolitons. New developments in Lie theory and geometry, Contemp. Math., 491, Amer. Math. Soc., Providence, RI, 2009, 1-35.

[27] J. Lauret, The Ricci flow for simply connected nilmanifolds, Preprint at arXiv:1004.0946v1 [math.DG].

[28] J. Lauret, Homogeneous Ricci flows and solitons. Preprint.

[29] J. Lott, On the long-time behavior of type-III Ricci flow solutions, Math. Ann. 339 (2007), 627-666.

[30] J. Lott, Dimensional reduction and the long-time behavior of Ricci flow, Comment. Math. Helv. 85(3) (2010), 485-534.

[31] J. Milnor, Curvatures of left invariant metrics on Lie groups, Adv. Math. 21(3) (1976), 293-329.

[32] T. Payne, The existence of soliton metrics for nilpotent Lie groups, Geom. Dedicata 145 (2010), 71-88. 
[33] T. Payne, The Ricci flow for nilmanifolds, J. Mod. Dyn. 4(1) (2010), 65-90.

[34] L. Perko, Differential equations and dynamical systems, 2nd edition. Texts in Applied Mathematics, 7, Springer-Verlag, New York, 1996, xiv +519 .

[35] W.-X. Shi, Ricci deformation of the metric on complete noncompact Riemannian manifolds, J. Differential Geom. 30(2) (1989), 303-394.

[36] W.-X. Shi, Complete noncompact three-manifolds with nonnegative Ricci curvature. J. Differential Geom. 29(2) (1989), 353-360.

Department of Mathematics

UNIVERSITY OF ARIZONA

TUCSON AZ 85721

USA

Department of MAThematics

IDAHO STATE UNIVERSITY

POCATELLO ID 83209-8085

USA

E-mail address: glickenstein@math.arizona.edu

E-mail address: payntrac@isu.edu, tpayne@member.ams.org

RECEIVED MAY 28, 2010 
